(2) Open Access Full Text Article

\title{
Potential role for mammalian target of rapamycin inhibitors as first-line therapy in hormone receptor-positive advanced breast cancer
}

This article was published in the following Dove Press journal:

OncoTargets and Therapy

7 December 2015

Number of times this article has been viewed

\author{
J Thaddeus Beck \\ Highlands Oncology Group, \\ Fayetteville, AR, USA
}

Correspondence: J Thaddeus Beck Highlands Oncology Group, 3232 N North Hills Blvd, Fayetteville, AR 72703, USA

Tel + I 479587 I 700

Email tbeck@hogonc.com

\begin{abstract}
Despite advances in cytotoxic chemotherapy and targeted therapies, 5-year survival rates remain low for patients with advanced breast cancer at diagnosis. This highlights the limited effectiveness of current treatment options. An improved understanding of cellular functions associated with the development and progression of breast cancer has resulted in the creation of a number of novel targeted molecular therapies. However, more work is needed to improve outcomes, particularly in the first-line recurrent or metastatic hormone receptor-positive breast cancer setting. The phosphatidylinositol 3-kinase/protein kinase B/mammalian target of rapamycin (mTOR) pathway is a major intracellular signaling pathway that is often upregulated in breast cancer, and overactivation of this pathway has been associated with primary or developed resistance to endocrine treatment. Clinical data from the Phase III Breast Cancer Trials of Oral Everolimus-2 (BOLERO-2) study of the mTOR inhibitor everolimus combined with exemestane in hormone receptor-positive advanced breast cancer were very promising, highlighting the potential role of mTOR inhibitors in combination with endocrine therapies as a first-line treatment option for these patients. It is hoped that the use of mTOR inhibitors combined with current standardof-care endocrine therapies, such as aromatase inhibitors, in the first-line advanced breast cancer setting may result in greater antitumor effects and also delay or reverse treatment resistance.
\end{abstract}

Keywords: mammalian target of rapamycin, everolimus, hormone receptor-positive breast cancer, first-line

\section{Introduction}

In the US, breast cancer is the most common type of cancer and the second leading cause of cancer mortality among women. ${ }^{1,2}$ Approximately one in eight women will be diagnosed with breast cancer during her lifetime. ${ }^{3,4}$ In 2015 , there will be an estimated 231,840 new cases of breast cancer diagnosed in women in the US, and it is predicted that 40,290 women will die of their disease. ${ }^{1}$ Unfortunately, most breast cancers are invasive at diagnosis, ${ }^{3}$ and approximately $5 \%$ to $10 \%$ of women have metastatic disease at the time of diagnosis. ${ }^{5}$ Additionally, up to $30 \%$ of those with lymph node-negative (ie, node-negative) and up to $70 \%$ of those with node-positive early-stage breast cancer can be expected to relapse. ${ }^{5}$

Despite advances in cytotoxic chemotherapy and targeted therapies, 5-year survival rates remain low ( $25 \%$ for patients with distant disease at diagnosis $),{ }^{4}$ highlighting the limited effectiveness of current treatment options, particularly in those with advanced breast cancer. The majority of advanced breast cancers are considered incurable. ${ }^{5}$ Tumor stage, tumor grade, hormone receptor (HR; ie, estrogen receptor [ER] or progesterone receptor), and human epidermal growth factor receptor 2 (HER2) status 
are important factors that guide treatment decisions. ${ }^{6} \mathrm{~A}$ better understanding of the underlying mechanisms involved in breast cancer growth and metastasis (eg, cell signaling pathways, DNA repair, angiogenesis) has led to the development of targeted therapies such as endocrine and biologic therapies, which include tamoxifen, aromatase inhibitors (AIs), trastuzumab, pertuzumab, trastuzumab emtansine, phosphatidylinositol 3-kinase (PI3K)/mammalian target of rapamycin (mTOR) dual inhibitor, and cyclin-dependent kinase (CDK) inhibitors; ${ }^{7-9}$ the increased use of these therapies in recent years has resulted in improvements in treatment outcomes. This review will discuss the current role of targeted therapies in the management of postmenopausal women with HR-positive advanced breast cancer and focus on the potential role of mTOR inhibitors in the first-line setting.

\section{Adjuvant versus first-line therapy}

Adjuvant therapy refers to the use of radiation therapy or systemic drug therapy (ie, chemotherapy, endocrine, and targeted therapy) following surgery to prevent disease recurrence. ${ }^{1,3,6}$ The use of systemic therapy in the adjuvant setting is primarily based on tumor stage and histopathological characteristics such as HR and HER2 status. ${ }^{6}$ Systemic therapy may also be considered as neoadjuvant therapy to shrink the primary tumor sufficiently to allow for surgical removal. ${ }^{3}$ Patients who present with metastatic disease may be unlikely to benefit from surgery or radiation therapy of the primary tumor. ${ }^{6}$ As a result, first-line (ie, initial) systemic therapy is often the preferred treatment option for women with advanced breast cancer. ${ }^{3}$ Given that most cases of advanced breast cancer are considered to be incurable, the management goals are usually to prolong survival and enhance quality of life. ${ }^{6}$ Therefore, first-line treatment options with minimal toxicity, such as endocrine therapies, are preferred over cytotoxic therapies. ${ }^{6}$

The National Comprehensive Cancer Network (NCCN) has indicated that the identification of contributing factors to therapeutic response (eg, HR, HER2) is a central component of the management of advanced breast cancer. ${ }^{6}$ It is important to note that subsequent therapy in patients whose disease has recurred or metastasized following adjuvant therapy is still considered first-line therapy (ie, first-line therapy in the recurrent or metastatic setting). Indeed, many clinical trials of first-line therapy in the recurrent or metastatic setting have allowed the inclusion of patients who have received previous adjuvant endocrine therapy. ${ }^{10-12}$

\section{Current first-line treatment options in advanced breast cancer}

The NCCN has established first-line treatment options for advanced metastatic or recurrent breast cancer. ${ }^{6}$ Women with ER-positive and/or progesterone receptor-positive advanced metastatic or recurrent breast cancer are good candidates for endocrine therapy, which is associated with relatively low toxicity profiles. ${ }^{6}$ However, a recent study of adherence to guidelines by the NCCN found that almost two-thirds of patients with advanced HR-positive, HER2-negative breast cancer had chemotherapy initiated without any prior endocrine treatment. ${ }^{13}$

Selection of first-line agents is based on numerous factors, including menopausal status, time since original diagnosis, and the prior use of adjuvant therapy for the primary tumor. ${ }^{6}$ For premenopausal women, available endocrine therapies include selective ER modulators (tamoxifen or toremifene), luteinizing hormone-releasing hormone agonists (goserelin or leuprolide), high-dose estrogen (ethinyl estradiol), androgens (fluoxymesterone), or progestin (megestrol acetate). In most premenopausal women who have received prior treatment with tamoxifen, additional endocrine therapy with or without ovarian suppression or ablation is appropriate. ${ }^{6}$ In postmenopausal women, endocrine therapies may include third-generation nonsteroidal (anastrazole and letrozole) and steroidal (exemestane) AIs, selective ER modulators, ER downregulators (fulvestrant), high-dose estrogen, androgens, or progestin. ${ }^{6}$ AIs have demonstrated superiority over tamoxifen in terms of response rate, clinical benefit rate, and progression-free survival (PFS), although the difference in survival benefit does not appear to be significant. ${ }^{14}$ However, tamoxifen is still an acceptable first-line option. ${ }^{6}$

According to NCCN guidelines, the selected endocrine therapy should be continued until disease progression or unacceptable toxicity, after which a new endocrine therapy can be selected for patients who derive clinical benefit after three sequential endocrine regimens or for those with no symptomatic visceral disease. ${ }^{6}$ Chemotherapy should be considered in patients who have HR-negative tumors that are not localized only to the bone or soft tissue and who have evidence of visceral crisis, or for those who have HR-positive tumors that are refractory to endocrine therapy. ${ }^{6}$ For patients with bone disease, denosumab or zoledronic acid should be added to the treatment course recommended for patients without bone disease. ${ }^{6}$

Resistance to endocrine therapy and cytotoxic therapy in breast cancer has been associated with activation of the $\mathrm{PI} 3 \mathrm{~K} /$ protein kinase B (Akt)/mTOR signaling pathway. ${ }^{15-17}$ 
The activation of cell signaling pathways (eg, the PI3K/ Akt/mTOR pathway) may mediate endocrine resistance. ${ }^{17}$ As a result, targeting therapeutics to this pathway may improve response in patients receiving an AI. Three mTOR inhibitors - temsirolimus, everolimus, and sirolimus - have been evaluated in breast cancer, but only everolimus has demonstrated favorable results. ${ }^{12,18,19}$ The Phase III Breast Cancer Trials of Oral Everolimus-2 (BOLERO-2) trial indicated that everolimus plus exemestane significantly prolonged PFS compared with exemestane alone in women with HR-positive, HER2-negative advanced breast cancer who had disease progression or recurrence on prior nonsteroidal AI (NSAI) therapy. ${ }^{12,18}$ Based on this evidence, the NCCN panel unanimously agreed that the addition of everolimus to exemestane may be considered in women who fulfill the entry criteria for BOLERO- $2{ }^{6}$

\section{Molecular targeted therapy: role of the PI3K/Akt/mTOR pathway in advanced breast cancer}

$\mathrm{PI} 3 \mathrm{~K} / \mathrm{Akt} / \mathrm{mTOR}$ is a major intracellular signaling pathway that regulates the proliferation, metabolism, motility, angiogenesis, and survival of normal cells and cancer cells. ${ }^{16,20-22}$ The PI3K heterodimer plays a central role in this pathway and is activated by a variety of receptor tyrosine kinases, including epidermal growth factor receptor, insulin-like growth factor 1 receptor (IGF1R), insulin receptor, and HER2. ${ }^{16,20-22}$ The activation of PI3K results in the downstream phosphorylation of Akt, a serine/threonine kinase, which in turn activates additional key downstream effectors, including mTOR..$^{22}$ mTOR is a serine/threonine protein kinase that regulates cell proliferation, apoptosis, angiogenesis, and cellular metabolism through two multiprotein complexes: mTOR complex 1 (mTORC1), which is sensitive to rapamycin inhibition, and $\mathrm{mTORC} 2 .{ }^{23} \mathrm{mTORC} 1$ enhances mRNA translation and cell proliferation, growth, and survival through phosphorylation of the eukaryotic initiation factor 4E-binding protein 1 and the p70 ribosomal S6 kinase 1.23,24 The function of mTORC2 is not as well understood but may be associated with cytoskeleton organization and cell proliferation, survival, and metabolism..$^{23,24}$

Dysregulation of the PI3K/Akt/mTOR pathway occurs in many tumor types, including breast cancer, and is associated with cancer pathogenesis, progression, and treatment resistance. ${ }^{17,25-27}$ Mutations in the PI3K catalytic alpha subunit and the loss of the tumor suppressor phosphatase and tensin homolog are frequently encountered in breast cancer. ${ }^{28} \mathrm{PI} 3 \mathrm{~K} /$ Akt activation in breast cancer cells and high levels of Akt or mTOR activity are associated with the development of resistance to endocrine therapy: ${ }^{17,25-27} \mathrm{PI} 3 \mathrm{~K}$ activation has also been associated with trastuzumab resistance. ${ }^{29-31}$ Taken together, this knowledge provides the rationale for the use of inhibitors of the PI3K/Akt/mTOR pathway in the treatment of patients with advanced breast cancer, thereby potentially delaying or reversing resistance to endocrine therapies.

\section{Current clinical data available for inhibitors of the PI3K/Akt/mTOR pathway}

The clinical data available to date indicate that mTOR inhibition may play a significant role in the treatment of HR-positive tumors. ${ }^{12,18}$ Several clinical trials have evaluated mTOR inhibitors as first-line therapy in HR-positive advanced breast cancer, ${ }^{12}$ but so far everolimus remains the only mTOR inhibitor that has been approved by the US Food and Drug Administration (FDA). Everolimus, in combination with exemestane, has been indicated for the treatment of postmenopausal women with HR-positive advanced breast cancer who have progressed after NSAI therapy. ${ }^{32}$ This approval, in 2012, was based on the findings of the randomized Phase III BOLERO-2 trial, which evaluated the addition of everolimus to exemestane in postmenopausal women with ER-positive, HER2-negative advanced breast cancer who had relapsed or progressed on NSAIs in the adjuvant or metastatic setting. ${ }^{12} \mathrm{~A}$ significant improvement in median PFS (central assessment) was seen with everolimus plus exemestane versus exemestane alone (11.0 vs 4.1 months; hazard ratio, $0.38 ; 95 \%$ confidence interval [CI], 0.31-0.48; $P<0.001) .{ }^{12}$ A final PFS analysis of the study confirmed this benefit in the overall population and prospectively defined subgroups. ${ }^{18}$ Everolimus plus exemestane was, however, associated with a higher incidence of adverse events (AEs) and toxicity-related treatment discontinuation $(26.3 \%$ for the everolimus and exemestane combination and $5 \%$ for exemestane alone). The most commonly reported AEs ( $>25 \%$ patients) were stomatitis, rash, and fatigue. ${ }^{18}$

A recent exploratory analysis of the BOLERO-2 trial was conducted in the subgroup of patients who had disease recurrence during or after neoadjuvant and adjuvant NSAI therapy or adjuvant AI therapy only. ${ }^{33}$ Based on local investigator assessment, the improvement in PFS with everolimus plus exemestane versus placebo plus exemestane was nearly tripled (11.5 vs 4.1 months; hazard ratio, $0.39 ; 95 \%$ CI, 0.25-0.62). ${ }^{33}$ This was confirmed by central assessment (15.2 vs 4.2 months; hazard ratio, 0.32 ; $95 \%$ CI, $0.18-0.57$ ). ${ }^{33}$ A retrospective, exploratory biomarker analysis indicates no predictive marker for everolimus efficacy within the four 
most frequently altered genes/pathways (PI3K, CCND1, phosphatase and tensin homolog [PTEN], and fibroblast growth factor receptor $1 / 2$ ) in the BOLERO-2 patient population. ${ }^{34}$ In this analysis, patients with wild-type genes or a single genetic alteration in PIK3CA, PTEN, CCND1, or fibroblast growth factor receptor $1 / 2$ genes derived greater benefit with everolimus compared with the overall population, whereas patients with genetic alterations in at least two of these genes still benefited from the addition of everolimus but to a lesser degree compared with the single/no mutation group. Elsewhere, in an exploratory analysis of the Phase II, open-label Tamoxifen Plus Everolimus (TAMRAD) study in patients with advanced breast cancer, there was a positive correlation between late effectors of mTORC1 activation and Akt-independent mTORC1 activation and everolimus efficacy, and an inverse correlation between canonical PI3K/ Akt/mTOR pathway and everolimus efficacy. ${ }^{35}$ Further prospective study is required to validate the clinical implications of these observations. These findings further support the use of everolimus in the first-line setting and highlight the potential benefit of early introduction of everolimus plus exemestane in the treatment of ER-positive, HER2-negative advanced breast cancer. ${ }^{33}$

The results of the BOLERO-2 exploratory analysis are supported by an earlier Phase II, randomized, placebocontrolled study that assessed neoadjuvant everolimus plus letrozole versus letrozole alone in patients with ER-positive breast cancer. ${ }^{36}$ After 4 months of neoadjuvant treatment, response rate by clinical palpation (primary end point) was higher with everolimus plus letrozole versus letrozole plus placebo (68.1\% vs 59.1\%; $P=0.0616$ [significance threshold, $P \leq 0.10]) .{ }^{36}$ The response rate by ultrasound was significantly improved with everolimus plus letrozole (58.0\% vs $47.0 \%$; $P=0.0352) .{ }^{36}$ Everolimus plus letrozole was associated with an acceptable safety profile with stomatitis being the mostly reported common side effect (36.5\%). Everolimus toxicity in this study was consistent with historical data. ${ }^{36}$ Based on these results, everolimus appears to have the potential to enhance the efficacy of letrozole when initiated early (ie, in the neoadjuvant setting) in patients with ER-positive breast cancer.

The TAMRAD trial assessed everolimus plus tamoxifen in postmenopausal women with HR-positive, HER2-negative advanced breast cancer who had progressed on previous AI therapy in the adjuvant or metastatic setting. ${ }^{37}$ In the overall study population, $41 \%$ of patients in the adjuvant setting and $67 \%$ in the first-line metastatic setting had received previous AI therapy. ${ }^{37}$ However, because some of the patients had received both adjuvant and first-line systemic therapy, only some of them could be considered first-line for the purposes of this study. A significant improvement in clinical benefit rate (primary end point) was seen with everolimus plus tamoxifen versus tamoxifen alone ( $61 \%$ vs $42 \% ; P=0.045)$; in addition, there was an improvement in time to progression (8.6 vs 4.5 months; $P=0.002)$ and a $55 \%$ reduction in the risk of death with combination therapy $(P=0.007) .{ }^{37}$ The most commonly reported nonhematologic toxicities were pain $(86 \%)$, fatigue $(53 \%)$, stomatitis (7\%), rash (7\%), anorexia (18\%), and diarrhea $(11 \%) ; 35 \%$ of patients required an everolimus dose reduction or discontinued therapy because of side effects. ${ }^{37}$

Recently, a Phase II trial investigated the effects of combination therapy comprising everolimus and fulvestrant (an ER downregulator) in patients with ER-positive metastatic breast cancer who had progressed or relapsed on an AI within 6 months of enrollment. ${ }^{38}$ At study entry, $26 \%$ of patients had received prior AI therapy in the adjuvant setting and $74 \%$ had received it in the metastatic setting. ${ }^{38}$ Time to progression (primary end point) was 7.4 months; the objective response and clinical benefit rates were $13 \%$ and $55 \%$, respectively, following treatment with this combination therapy. ${ }^{38}$ The most common AEs were elevated aspartate aminotransferase (87\%), elevated alanine aminotransferase (77\%), anemia (74\%), hyperglycemia (71\%), and hypercholesteremia (68\%). ${ }^{38}$ AEs were mostly mild to moderate and reversible and everolimus dose reductions were not frequently required. ${ }^{38}$

In contrast to the findings of BOLERO-2, the randomized Phase III trial of first-line oral temsirolimus plus letrozole in patients with locally advanced or metastatic, AI inhibitor-naïve, ER-positive breast cancer did not demonstrate improvements in response rates or PFS versus letrozole alone. ${ }^{19}$ No overall improvement in median PFS (primary end point) was seen between temsirolimus plus letrozole and letrozole alone ( 8.9 vs 9.0 months; hazard ratio, $0.90 ; P=0.25) .{ }^{19}$ Grade 3 to 4 AEs were more frequently reported with temsirolimus plus letrozole (37\% vs $24 \%){ }^{19}$ An exploratory analysis showed that PFS was longer with letrozole plus temsirolimus versus letrozole alone in younger patients aged $\leq 65$ years (9.0 vs 5.6 months; hazard ratio, $0.75 ; 95 \%$ CI, 0.60-0.93; $P=0.009) .{ }^{19}$ However, the lack of overall PFS benefit with the letrozole plus temsirolimus combination was surprising and is difficult to explain. It is possible that the difference in PFS outcomes between the temsiroliums plus letrozole trial and BOLERO-2 reflects key differences in patient characteristics between the two trials. For example, the temsirolimus plus letrozole trial excluded patients with prior exposure to AIs, ${ }^{19}$ whereas a selection criterion in BOLERO-2 was progression on an NSAI during or within 12 months of adjuvant therapy, or progression within 1 month in the metastatic setting. ${ }^{12,18}$ 
There are currently no FDA-approved PI3K or Akt inhibitors, although various clinical trials evaluating inhibitors of the PI3K/Akt/mTOR pathway in HR-positive advanced breast cancer are ongoing (Table 1). There are also a number of agents in development that may offer dual PI3K-mTOR inhibition. Dual inhibition is being investigated because inhibition of mTORC1 activity alone may lead to the enhanced activation of the PI3K axis, and it is thought that dual inhibition of PI3K-mTOR or mTORC1-mTORC2 may be sufficient to avoid PI3K reactivation. ${ }^{7}$ In theory, dual inhibition targets PI3K, mTORC1, and mTORC2, efficiently shutting down the PI3K/Akt/mTOR pathway. ${ }^{7}$ However, targeting multiple points in this pathway also has the potential to result in enhanced toxicity.

Of particular note among the trials targeting the PI3K/Akt/ mTOR pathway is the multicenter, open-label, single-arm,
Phase II BOLERO-4 trial that is currently being conducted at sites across Europe, Asia, and the Americas. ${ }^{39}$ BOLERO-4 aims to assess the safety and effectiveness of first-line therapy with everolimus plus letrozole in postmenopausal patients with locally advanced or metastatic ER-positive, HER2negative breast cancer. ${ }^{39}$ It is hoped that this study will provide insight into the efficacy of first-line combination therapy with everolimus plus letrozole in patients with metastatic breast cancer, and insight into the efficacy of continued everolimus following initial disease progression; patients who progress during treatment with everolimus plus letrozole will be permitted to receive everolimus plus exemestane until further disease progression or unacceptable toxicity. ${ }^{39}$ The study is using PFS as the primary end point. It has completed enrollment $(\mathrm{N}=202)$ and has an estimated study-completion date of December 2016 (https://clinicaltrials.gov/ct2/show/NCT01698918).

Table I Ongoing clinical trials investigating inhibitors of the PI3K/Akt/mTOR pathway in HR-positive advanced breast cancer

\begin{tabular}{|c|c|c|c|c|}
\hline Therapeutic target & Agent & $\begin{array}{l}\text { ClinicalTrials.gov } \\
\text { identifier }\end{array}$ & Status & Study details \\
\hline \multirow[t]{3}{*}{ mTOR inhibitors } & Everolimus & NCT0I698918 & $\begin{array}{l}\text { Active, not } \\
\text { recruiting }\end{array}$ & $\begin{array}{l}\text { Phase II; everolimus + letrozole, everolimus + exemestane } \\
\text { (offered if patients progress); locally advanced or metastatic } \\
\text { ER-positive/HER2-negative BC }\end{array}$ \\
\hline & Everolimus & NCT0I 783444 & $\begin{array}{l}\text { Active, not } \\
\text { recruiting }\end{array}$ & $\begin{array}{l}\text { Phase II; everolimus, capecitabine, or } \\
\text { everolimus + exemestane; ER-positive advanced BC }\end{array}$ \\
\hline & Ridaforolimus & NCT0I605396 & $\begin{array}{l}\text { Active, not } \\
\text { recruiting }\end{array}$ & $\begin{array}{l}\text { Phase II; ridaforolimus + exemestane or ridaforolimus + } \\
\text { dalotuzumab + exemestane; ER-positive/HER2-negative locally } \\
\text { advanced or metastatic BC }\end{array}$ \\
\hline \multirow[t]{6}{*}{ Pan-PI3K inhibitors } & BKMI20 & NCT0I633060 & Recruiting & $\begin{array}{l}\text { Phase III; BKMI } 20 \text { + fulvestrant or BKMI } 20 \text { + placebo; } \\
\text { Al-treated, locally advanced or metastatic BC progressing on } \\
\text { or after prior treatment with an mTOR inhibitor + endocrine } \\
\text { therapy }\end{array}$ \\
\hline & BKMI20 & NCT0I572727 & $\begin{array}{l}\text { Active, not } \\
\text { recruiting }\end{array}$ & $\begin{array}{l}\text { Phase II/III; BKMI } 20 \text { + paclitaxel or placebo + paclitaxel; } \\
\text { HER2-negative, locally advanced or metastatic BC with or } \\
\text { without PI3K activation }\end{array}$ \\
\hline & BKMI20 & NCT0I6I0284 & $\begin{array}{l}\text { Active, not } \\
\text { recruiting }\end{array}$ & $\begin{array}{l}\text { Phase III; BKMI } 20 \text { + fulvestrant or placebo + fulvestrant; } \\
\text { HR-positive/HER2-negative locally advanced or metastatic BC } \\
\text { refractory to Al therapy }\end{array}$ \\
\hline & GDC-094I & NCT0I437566 & $\begin{array}{l}\text { Active, not } \\
\text { recruiting }\end{array}$ & $\begin{array}{l}\text { Phase II; GDC-094I + fulvestrant or GDC-0980 + fulvestrant } \\
\text { or placebo + fulvestrant; HR-positive/HER2-negative locally } \\
\text { advanced or metastatic BC progressing on or after Al therapy }\end{array}$ \\
\hline & GDC-094I & NCT0I740336 & $\begin{array}{l}\text { Active, not } \\
\text { recruiting }\end{array}$ & $\begin{array}{l}\text { Phase II; GDC-094I + paclitaxel or placebo + paclitaxel; } \\
\text { HR-positive/HER2-negative locally recurrent or metastatic BC }\end{array}$ \\
\hline & GDC-094I & NCT00960960 & $\begin{array}{l}\text { Active, not } \\
\text { recruiting }\end{array}$ & $\begin{array}{l}\text { Phase I; GDC-094I + paclitaxel } \pm \text { bevacizumab or } \\
\text { trastuzumab; locally recurrent or metastatic BC }\end{array}$ \\
\hline Akt inhibitors & MK2206 & NCT0I776008 & Recruiting & $\begin{array}{l}\text { Phase II; MK2206 + anastrozole } \pm \text { goserelin acetate; } \\
\text { ER-positive/HER2-negative nonmetastatic invasive BC }\end{array}$ \\
\hline \multirow[t]{2}{*}{$\mathrm{mTORCI} / \mathrm{mTORC2}$} & AZD20I4 & NCT0I597388 & Recruiting & Phase I; AZD20I4 + fulvestrant; ER-positive advanced MBC \\
\hline & AZD2014 & NCT02216786 & Recruiting & $\begin{array}{l}\text { Phase II; AZD20I4 + fulvestrant, everolimus + fulvestrant; } \\
\text { ER-positive metastatic BC }\end{array}$ \\
\hline
\end{tabular}

Abbreviations: Al, aromatase inhibitor; Akt, protein kinase B; BC, breast cancer; ER, estrogen receptor; HER2, human epidermal growth factor receptor 2; HR, hormone receptor; MBC, metastatic breast cancer; mTOR, mammalian target of rapamycin; PI3K, phosphatidylinositol 3-kinase. 


\section{Overview of other targets being investigated}

There are a number of therapeutic targets other than the PI3K/ Akt/mTOR pathway that are being evaluated in the setting of HR-positive advanced breast cancer and include aspects of cell cycle control, the IGF1R, farnesyltransferase, vascular endothelial growth factor (VEGF), and the Src oncogene (Figure 1). ${ }^{40}$ Because many of these targets are in the early phases of clinical investigation, most studies mentioned in the next section (particularly those in early phases) included a mix of patients who had received prior therapy in the adjuvant and/or metastatic setting; not many were conducted in a purely first-line metastatic setting. Table 2 summarizes clinical trials evaluating select targeted therapies in HR-positive advanced breast cancer.

Loss of cell cycle control is seen in many cancers, including breast cancer, and cyclin activation of CDKs is pivotal for the control of cell cycle progression. ${ }^{41}$ In particular, CDK 4/6 activation mediates the phosphorylation of retinoblastoma tumor-suppressor protein resulting in the activation of E2F transcription factors, G1/S transition, and cell cycle progression. ${ }^{7}$ Palbociclib (PD-0332991), a first-in-class selective CDK4/6 inhibitor, has been shown to be an effective treatment for ER-positive, HER2-negative metastatic breast cancer. ${ }^{42}$ A Phase II clinical trial investigating palbociclib in heavily pretreated patients $(76 \%$ patients $\geq 2$ lines of therapy) with retinoblastoma-positive breast cancer showed clinical benefit in $21 \%$ of ER-positive patients. ${ }^{43}$ The median PFS for patients with ER-positive, HER2-negative and ER-positive, HER2-positive disease was 3.8 and 5.1 months, respectively. ${ }^{43}$ None of the biomarkers analyzed in the study were significantly associated with clinical benefit or PFS. ${ }^{43}$ A randomized, Phase II study evaluated first-line treatment of palbociclib in combination with letrozole versus letrozole alone in patients with ER-positive, HER2-negative advanced breast cancer. ${ }^{44}$ There was a significant improvement in median PFS with palbociclib plus letrozole versus letrozole alone (20.2 vs 10.2 months; hazard ratio, $0.49 ; 95 \% \mathrm{CI}, 0.32-0.75 ; P=0.0004) .{ }^{44}$ However, analysis of cyclin D1 and p16 as biomarkers in this study revealed the benefit from palbociclib and letrozole was less in the biomarker-enriched cohort, suggesting

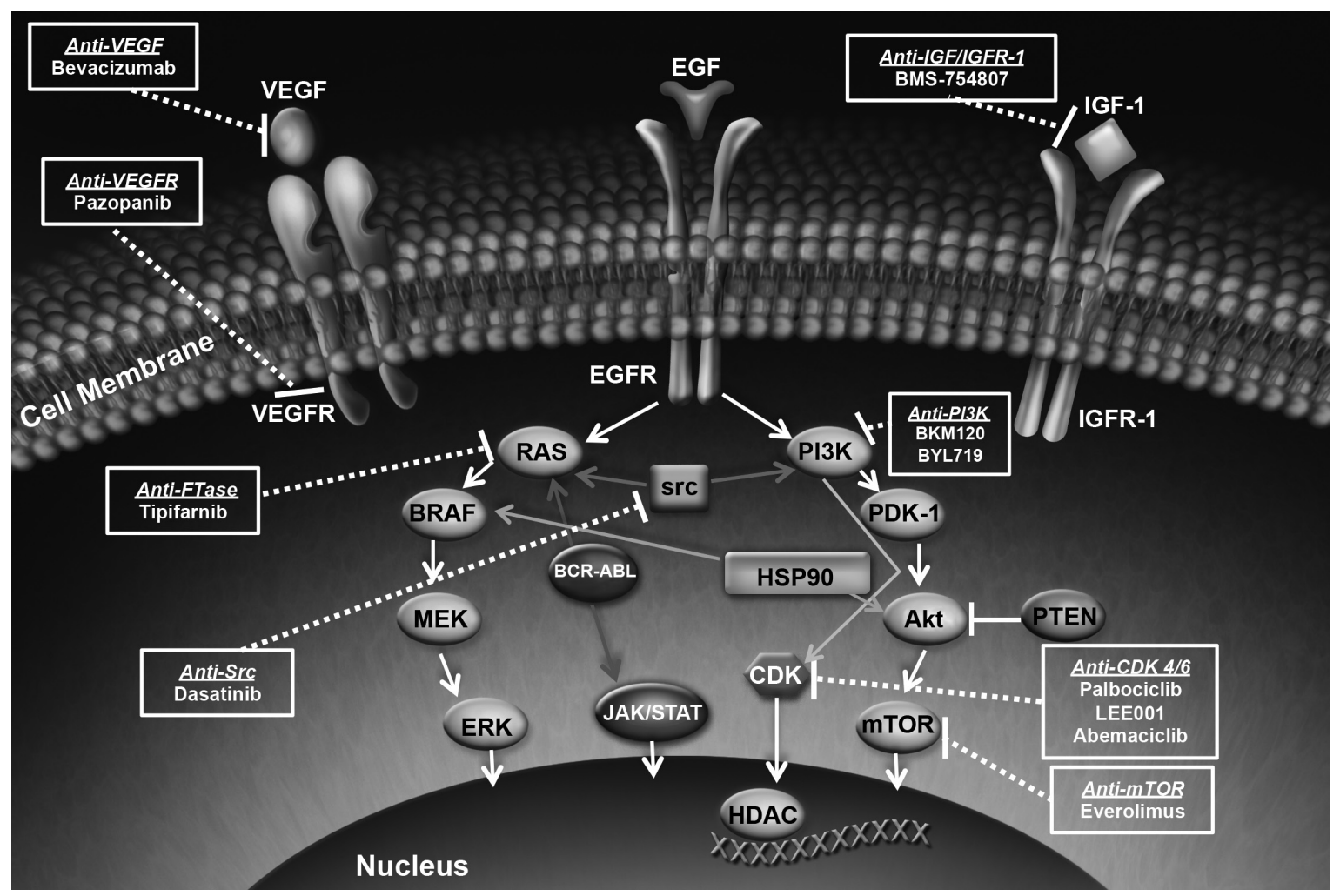

Figure I Overview of select targeted agents in the treatment of advanced breast cancer. Adapted from Munagala R, Aqil F, Gupta RC. Promising molecular targeted therapies in breast cancer. Indian J Pharmacol. 201 I;43(3):236-245.40

Abbreviations: Akt, protein kinase B; BCR-ABL, Philadelphia chromosome; BRAF, B-type RAF kinase; CDK, cyclin-dependent kinase; EGF, epidermal growth factor; EGFR, EGF receptor; ERK, extracellular signal-regulated kinase; FTase, farnesyltransferase; HDAC, histone deacetylase; HSP90, heat shock protein 90; IGF-I, insulin-like growth factor I; IGFR-I, IGF-I receptor; JAK/STAT, Janus kinases/signal transducers and activators of transcription; MEK, mitogen-activated protein kinase kinase; mTOR, mammalian target of rapamycin; PDK-I, pyruvate dehydrogenase kinase isozyme I; PI3K, phosphatidylinositol 3-kinase; PTEN, phosphatase and tensin homolog; Ras, Rat sarcoma subfamily of GTPases; src, v-Src (Rous sarcoma virus) tyrosine kinase; VEGF, vascular endothelial growth factor; VEGFR, VEGF receptor. 
Table 2 Clinical trials investigating select targeted therapies other than inhibitors of the PI3K/Akt/mTOR pathway in HR-positive advanced breast cancer

\begin{tabular}{|c|c|c|c|c|}
\hline Therapeutic target & Agent & $\begin{array}{l}\text { ClinicalTrial.gov } \\
\text { identifier }\end{array}$ & Status & Study details \\
\hline \multirow[t]{13}{*}{ CDK 4/6 inhibitors } & Palbociclib & NCT02028507 & Recruiting & $\begin{array}{l}\text { Phase III; palbociclib + exemestane, or single-agent capecitabine; } \\
\text { HR-positive/HER2-negative metastatic BC resistant to NSAI } \\
\text { therapy }\end{array}$ \\
\hline & Palbociclib & NCT0I740427 & $\begin{array}{l}\text { Active, not } \\
\text { recruiting }\end{array}$ & $\begin{array}{l}\text { Phase III; palbociclib + letrozole or placebo + letrozole; ER- } \\
\text { positive/HER2-negative advanced BC }\end{array}$ \\
\hline & Palbociclib & NCT01942I35 & $\begin{array}{l}\text { Active, not } \\
\text { recruiting }\end{array}$ & $\begin{array}{l}\text { Phase III; palbociclib + fulvestrant or placebo + fulvestrant; } \\
\text { HR-positive/HER2-negative metastatic BC following endocrine } \\
\text { treatment failure }\end{array}$ \\
\hline & Palbociclib & NCT0I6842I5 & Recruiting & $\begin{array}{l}\text { Phase II; palbociclib } \pm \text { letrozole; Japanese patients with } \\
\text { ER-positive/HER2-negative locally advanced or metastatic BC }\end{array}$ \\
\hline & Palbociclib & NCT0072I409 & $\begin{array}{l}\text { Active, not } \\
\text { recruiting }\end{array}$ & $\begin{array}{l}\text { Phase I/II; palbociclib + letrozole or single-agent letrozole; } \\
\text { ER-positive/HER2-negative ABC }\end{array}$ \\
\hline & LEEOII & NCT0I872260 & Recruiting & $\begin{array}{l}\text { Phase I/II; LEOII + letrozole, BYL7I9 + letrozole, or LE0II + } \\
\text { BYL7I9 + letrozole; ER-positive/HER2-negative, locally advanced } \\
\text { or metastatic BC }\end{array}$ \\
\hline & LEEOII & NCT02I 54776 & Recruiting & $\begin{array}{l}\text { Phase I; LEEOII + buparlisib + letrozole; HR-positive/HER2- } \\
\text { negative locally advanced or metastatic BC }\end{array}$ \\
\hline & LEEOII & NCT0I95802I & Recruiting & $\begin{array}{l}\text { Phase III; LEEOII + letrozole or placebo + } \\
\text { letrozole; HR-positive/HER2-negative, locally advanced or } \\
\text { metastatic BC with no prior treatment for metastatic disease }\end{array}$ \\
\hline & LEEOII & NCT0I857/93 & Recruiting & $\begin{array}{l}\text { Phase I/II; LEEOII + everolimus + exemestane, LEEOI I + } \\
\text { exemestane, or exemestane + everolimus; ER-positive/HER2- } \\
\text { negative, locally advanced or metastatic BC }\end{array}$ \\
\hline & LEEOII & NCT02278I20 & Recruiting & $\begin{array}{l}\text { Phase III; LEEOI I + NSAI + tamoxifen + goserelin, or placebo + } \\
\mathrm{NSAI}+\text { tamoxifen + goserelin; HR-positive/HER2-negative, } \\
\text { locally advanced or metastatic BC }\end{array}$ \\
\hline & Abemaciclib & NCT02057I33 & Recruiting & $\begin{array}{l}\text { Phase I; abemaciclib + hormone therapies (letrozole; } \\
\text { anastrozole; tamoxifen; exemestane; exemestane + everolimus; } \\
\text { trastuzumab); HR-positive/HER2-negative, metastatic BC }\end{array}$ \\
\hline & Abemaciclib & NCT0224662I & Recruiting & $\begin{array}{l}\text { Phase III; abemaciclib + NSAI or placebo + NSAl; HR-positive/ } \\
\text { HER2-negative, previously untreated, locally advanced or } \\
\text { metastatic BC }\end{array}$ \\
\hline & Abemaciclib & NCT02107703 & Recruiting & $\begin{array}{l}\text { Phase III; fulvestrant } \pm \text { abemaciclib or placebo; HR-positive/ } \\
\text { HER2-negative, locally advanced or metastatic BC }\end{array}$ \\
\hline IGFIR inhibitors & BMS-754807 & NCT0I 225172 & Completed & $\begin{array}{l}\text { Phase II; BMS- } 754807 \pm \text { letrozole; HR-positive/HER2-negative, } \\
\text { locally advanced or metastatic BC progressing on prior NSAI } \\
\text { therapy }\end{array}$ \\
\hline FTase inhibitors & Tipifarnib & NCT00I00750 & Completed & Phase I/II; tipifarnib + gemcitabine; metastatic BC \\
\hline VEGFR inhibitors & Pazopanib & NCT0I466972 & Recruiting & $\begin{array}{l}\text { Phase II; pazopanib plus NSAI; ER-positive and/or PR-positive, } \\
\text { locally advanced or metastatic BC progressing during prior } \\
\text { hormonal therapy }\end{array}$ \\
\hline Src inhibitors & Dasatinib & NCT00696072 & $\begin{array}{l}\text { Active, not } \\
\text { recruiting }\end{array}$ & $\begin{array}{l}\text { Phase II; dasatinib + letrozole or single-agent letrozole; } \\
\text { HR-positive/HER2-negative, locally recurrent or metastatic BC }\end{array}$ \\
\hline
\end{tabular}

Abbreviations: BC, breast cancer; CDK, cyclin-dependent kinase; ER, estrogen receptor; FTase, farnesyltransferase; HER2, human epidermal growth factor receptor 2; HR, hormone receptor; IGFIR, insulin-like growth factor I receptor; NSAI, nonsteroidal aromatase inhibitor; PR, progesterone receptor; VEGFR, vascular endothelial growth factor receptor.

these are not suitable biomarkers for palbociclib. ${ }^{44}$ The combination of palbociclib plus letrozole was generally well tolerated, and the most frequently reported treatmentrelated AE was neutropenia (20\%). ${ }^{44}$ These findings suggest that the addition of palbociclib to letrozole provides clinical benefit in the first-line metastatic breast cancer setting. Based on these data, palbociclib was recently approved by the FDA for first-line use in combination with letrozole in postmenopausal women with HR-positive metastatic breast cancer. ${ }^{45}$ Phase III studies of this combination are under way (Table 2) with further biomarker data expected to be reported. Recently, a Phase III clinical 
trial involving palbociclib in combination with fulvestrant for second-line treatment for HR-positive, HER2-negative breast cancer (PALbociclib: Ongoing trials in the Management of breast cAncer [PALOMA-3]) was stopped early because it met its efficacy end point. ${ }^{46}$ The median PFS was 9.2 months (95\% CI, 7.5-not estimable) for the palbociclib and fulvestrant combination and 3.8 months (95\% CI, 3.5-5.5) for the placebo and fulvestrant combination (hazard ratio, $0.42 ; 95 \% \mathrm{CI}, 0.32-0.56 ; P<0.001) .{ }^{46} \mathrm{~A}$ similar rate of discontinuation was observed for the palbociclib and placebo groups, and the most frequently reported $\mathrm{AE}$ was neutropenia (78.8\%). ${ }^{46}$ Other CDK 4/6 inhibitors, including abemaciclib (LY2835219) and ribociclib (LEE011), are also in clinical development for the first-line treatment of HR-positive advanced breast cancer in combination with NSAIs or fulvestrant (Table 2).

IGF1R is a tyrosine kinase receptor involved in the regulation of energy metabolism, body size, and longevity. ${ }^{47}$ Preclinical data suggest that activation of IGF1R signaling has been linked to trastuzumab resistance in breast cancer cells. ${ }^{48}$ Published data for IGF1R inhibitors are limited in the first-line metastatic HR-positive breast cancer setting, but ongoing trials are being conducted for BMS-754807, a dual IGF1R/insulin receptor tyrosine kinase inhibitor (NCT01225172, Table 2).

Farnesyltransferase catalyzes the modification of the Ras protein, a process that is essential for the mediation of downstream signaling effects. ${ }^{8}$ The farnesyltransferase inhibitor tipifarnib has been assessed in a number of Phase II studies in patients with metastatic breast cancer with limited evidence that it is an effective antitumor agent. ${ }^{49,50}$

VEGF has been implicated in the transitioning of a tumor to a "prevascular" phase (ie, one with increased growth and greater metastatic potential), which is a hallmark of the malignant process and thought to be stimulated by the increased expression of proangiogenic factors. ${ }^{51}$ Therefore, blocking the VEGF pathway has the potential to inhibit tumor angiogenesis and growth. Bevacizumab is a monoclonal antibody therapy that has been extensively studied as an adjunctive therapy in patients with advanced breast cancer in a number of large Phase III trials. ${ }^{52,53}$ Results to date have been relatively disappointing with limited improvements in PFS and overall survival. Currently, the pan-VEGF inhibitor pazopanib is being evaluated as an add-on therapy in a Phase II trial of patients with HR-positive, locally advanced or metastatic breast cancer who have progressed on NSAI therapy in the adjuvant or metastatic setting (NCT01466972, Table 2).
The Src oncogene, which regulates cell proliferation, differentiation, survival, motility, and angiogenesis, is a key messenger in many cellular pathways. ${ }^{8,54}$ Src plays a role in signaling and crosstalk between growth-promoting pathways, such as the ER and epidermal growth factor receptor family signaling pathways. ${ }^{8}$ A Phase II study of first- and secondline dasatinib plus letrozole is currently being conducted in patients with HR-positive, HER2-negative locally advanced or metastatic breast cancer (NCT00696072, Table 2).

\section{Further directions}

Increased understanding of the molecular pathology of breast cancer and the various cell signaling pathways involved has led to a dramatic increase in the list of potential targets for drug treatment. This has resulted in the development of many novel targeted molecular therapies that may eventually lead to improvements in clinical outcomes. However, there is an urgent need for novel treatment strategies in the metastatic setting because there are currently few options available to treat these patients.

There is also an unmet need for effective first-line treatment options in patients with HR-positive recurrent or metastatic breast cancer who have progressed on endocrine therapy (particularly AIs). Results from the Phase III BOLERO-2 trial demonstrated a clinically meaningful improvement in PFS with the addition of the mTOR inhibitor everolimus in this treatment setting. However, a number of questions remain unanswered. A statistically significant improvement in median overall survival was not observed with everolimus plus exemestane versus placebo plus exemestane (31.0 vs 26.6 months; hazard ratio, $0.89 ; 95 \% \mathrm{CI}, 0.73-1.10 ; P=0.1426){ }^{50}$ Therefore, it has not yet been demonstrated that the improvements in PFS will translate to an overall survival benefit. It is also not clear whether everolimus could be used as a first-line treatment in patients with HR-positive advanced breast cancer who have not been previously exposed to endocrine therapy.

Similar to endocrine resistance, the prolonged use of mTOR inhibitors may also result in feedback activation of PI3K, and this may lead to mTOR-independent cell cycle progression. To address this issue, focus should be placed on the clinical development of second-generation mTOR inhibitors, such as dual PI3K-mTOR or mTORC1-mTORC2 inhibitors. These agents have the potential to provide efficient and comprehensive inhibition of the PI3K/Akt/mTOR pathway. However, enhanced toxicity may be an issue. It remains to be seen whether these second-generation mTOR inhibitors will provide improved outcomes when compared with everolimusbased therapy in the first-line recurrent or metastatic setting. 
Additional research is also required to gain a better understanding of which patients will gain the most benefit from targeted molecular therapies in the first-line setting. The determination of patients who will gain optimal benefit from targeted therapy in the first-line setting remains a challenge. Therefore, the development of robust biomarkers to help predict therapeutic response will form an important component of the management of advanced breast cancer.

\section{Conclusion}

A better understanding of the underlying mechanisms involved in breast cancer development and progression has led to the development of many novel targeted molecular therapies. This in turn has led to improvements in PFS and other measures of antitumor activity for many patients. However, improving overall survival rates remains a significant challenge, and there remains an urgent need for effective targeted treatment strategies, particularly in the first-line recurrent or metastatic setting. Recent advances, such as the approval of palbociclib as a new first-line treatment option, will extend the therapeutic options available to patients with ER-positive breast cancer. The PI3K/Akt/mTOR pathway is a major intracellular signaling pathway that is often upregulated in breast cancer, and activation of this pathway is also associated with endocrine treatment resistance. Currently available Phase III clinical data (BOLERO-2) of the mTOR inhibitor everolimus in HRpositive advanced breast cancer are encouraging, highlighting the potential role of mTOR inhibitors (in combination with endocrine therapies) as a first-line treatment option in patients with advanced breast cancer. The use of mTOR inhibitors in combination with standard-of-care endocrine therapies (eg, AI therapy) in the first-line advanced breast cancer setting may not only enhance the antitumor efficacy of these treatments but may also delay or reverse treatment resistance. Further clinical experience with everolimus and other agents, such as palbociclib, will provide greater insight into the relative benefits of every agent and their optimal clinical utility.

\section{Acknowledgments}

The author thanks Matthew Grzywacz, PhD, of ApotheCom (Yardley, PA, USA) for editorial and technical assistance in the development of this paper. Novartis Pharmaceuticals Corporation provided funding for the development of this paper.

\section{Disclosure}

Dr Beck has received research support from Genentech/ Roche, Eli Lilly, Amgen, Abbvie, Pfizer, and Novartis. The author reports no other conflicts of interest in this work.

\section{References}

1. American Cancer Society. Cancer Facts and Figures 2015. Atlanta, GA: American Cancer Society; 2015. Available from: http://www.cancer. org/acs/groups/content/@editorial/documents/document/acspc-044552. pdf. Accessed March 13, 2015.

2. Siegel R, Ma J, Zou Z, Jemal A. Cancer statistics, 2014. CA Cancer J Clin. 2014;64(1):9-29.

3. American Cancer Society. American Cancer Society Breast Cancer Facts and Figures 2013-2014. Atlanta, GA: American Cancer Society; 2013.

4. National Cancer Institute. SEER Statistics Fact Sheets: Breast. Bethesda, MD: National Cancer Institute; 2010. Available from: http://seer.cancer. gov/statfacts/html/breast.html\#survival. Accessed March 13, 2015.

5. Cardoso F, Harbeck N, Fallowfield L, Kyriakides S, Senkus E. Locally recurrent or metastatic breast cancer: ESMO Clinical Practice Guidelines for diagnosis, treatment and follow-up. Ann Oncol. 2012;23(Suppl 7): vii11-vii19.

6. National Comprehensive Cancer Network, Inc. NCCN Clinical Practice Guidelines in Oncology. Breast Cancer. version 2. Fort Washington, PA: National Comprehensive Cancer Network, Inc. 2015. Available from: http://www.nccn.org/patients. Accessed April 9, 2015.

7. Nwabo Kamdje AH, Seke Etet PF, Vecchio L, Muller JM, Krampera M, Lukong KE. Signaling pathways in breast cancer: therapeutic targeting of the microenvironment. Cell Signal. 2014;26(12):2843-2856.

8. de la Vega M, Diaz-Canton E, Alvarez RH. Novel targeted agents for the treatment of advanced breast cancer. Future Med Chem. 2012;4(7): 893-914.

9. Wicki A, Rochlitz C. Targeted therapies in breast cancer. Swiss Med Wkly. 2012;142:w13550.

10. Buzdar A, Jonat W, Howell A, et al. Anastrozole, a potent and selective aromatase inhibitor, versus megestrol acetate in postmenopausal women with advanced breast cancer: results of overview analysis of two phase III trials. Arimidex Study Group. J Clin Oncol. 1996;14(7): 2000-2011.

11. Buzdar A, Douma J, Davidson N, et al. Phase III, multicenter, doubleblind, randomized study of letrozole, an aromatase inhibitor, for advanced breast cancer versus megestrol acetate. J Clin Oncol. 2001;19(14): 3357-3366.

12. Baselga J, Campone M, Piccart M, et al. Everolimus in postmenopausal hormone receptor-positive advanced breast cancer. $N$ Engl $J$ Med. 2012;366(6):520-529.

13. Engel-Nitz NM, Hao Y, Willemann Rogerio J, et al. Hormone receptor positive $(\mathrm{HR}+) /$ human epidermal growth factor receptor 2 negative (HER2-) advanced breast cancer: sequencing of chemotherapy and endocrine therapy. Presented at: The American Society of Clinical Oncology Annual Meeting; May 31-June 4; 2013; Chicago, IL.

14. Gibson L, Lawrence D, Dawson C, Bliss J. Aromatase inhibitors for treatment of advanced breast cancer in postmenopausal women. Cochrane Database Syst Rev. 2009;(4):CD003370.

15. Nahta R. Pharmacological strategies to overcome HER2 cross-talk and trastuzumab resistance. Curr Med Chem. 2012;19(7):1065-1075.

16. Paplomata E, O'Regan R. New and emerging treatments for estrogen receptor-positive breast cancer: focus on everolimus. Ther Clin Risk Manag. 2013;9:27-36.

17. Musgrove EA, Sutherland RL. Biological determinants of endocrine resistance in breast cancer. Nat Rev Cancer. 2009;9(9):631-643.

18. Yardley DA, Noguchi S, Pritchard KI, et al. Everolimus plus exemestane in postmenopausal patients with $\mathrm{HR}(+)$ breast cancer: BOLERO-2 final progression-free survival analysis. Adv Ther. 2013;30(10):870-884.

19. Wolff AC, Lazar AA, Bondarenko I, et al. Randomized phase III placebo-controlled trial of letrozole plus oral temsirolimus as first-line endocrine therapy in postmenopausal women with locally advanced or metastatic breast cancer. J Clin Oncol. 2013;31(2):195-202.

20. Shaw RJ, Cantley LC. Ras, PI(3)K and mTOR signalling controls tumour cell growth. Nature. 2006;441(7092):424-430.

21. Laplante M, Sabatini DM. mTOR signaling in growth control and disease. Cell. 2012;149(2):274-293. 
22. Paplomata E, O'Regan R. The PI3K/AKT/mTOR pathway in breast cancer: targets, trials and biomarkers. Ther Adv Med Oncol. 2014;6(4): $154-166$.

23. Wullschleger $\mathrm{S}$, Loewith $\mathrm{R}$, Hall $\mathrm{MN}$. TOR signaling in growth and metabolism. Cell. 2006;124(3):471-484.

24. Guertin DA, Sabatini DM. Defining the role of mTOR in cancer. Cancer Cell. 2007;12(1):9-22.

25. deGraffenried LA, Friedrichs WE, Russell DH, et al. Inhibition of mTOR activity restores tamoxifen response in breast cancer cells with aberrant Akt activity. Clin Cancer Res. 2004;10(23):8059-8067.

26. Chollet $\mathrm{P}, \mathrm{Abrial} \mathrm{C}$, Tacca $\mathrm{O}$, et al. Mammalian target of rapamycin inhibitors in combination with letrozole in breast cancer. Clin Breast Cancer. 2006;7(4):336-338.

27. Beeram M, Tan QT, Tekmal RR, Russell D, Middleton A, deGraffenried LA. Akt-induced endocrine therapy resistance is reversed by inhibition of mTOR signaling. Ann Oncol. 2007;18(8):1323-1328.

28. Perez-Tenorio G, Alkhori L, Olsson B, et al. PIK3CA mutations and PTEN loss correlate with similar prognostic factors and are not mutually exclusive in breast cancer. Clin Cancer Res. 2007;13(12):3577-3584.

29. Nagata $Y$, Lan KH, Zhou X, et al. PTEN activation contributes to tumor inhibition by trastuzumab, and loss of PTEN predicts trastuzumab resistance in patients. Cancer Cell. 2004;6(2):117-127.

30. Berns K, Horlings HM, Hennessy BT, et al. A functional genetic approach identifies the PI3K pathway as a major determinant of trastuzumab resistance in breast cancer. Cancer Cell. 2007;12(4):395-402.

31. Kataoka Y, Mukohara T, Shimada H, Saijo N, Hirai M, Minami H. Association between gain-of-function mutations in PIK3CA and resistance to HER2-targeted agents in HER2-amplified breast cancer cell lines. Ann Oncol. 2010;21(2):255-262.

32. Afinitor (everolimus) tablets for oral administration [prescribing information]. Stein, Switzerland: Novartis Pharma Stein AG; April 2015.

33. Beck JT, Hortobagyi GN, Campone M, et al. Everolimus plus exemestane as first-line therapy in HR, HER2 advanced breast cancer in BOLERO-2. Breast Cancer Res Treat. 2014;143(3):459-467.

34. Hortobagyi G, Piccart M, Rugo H, et al. Genetic alterations and everolimus efficacy in hormone receptor-positive, HER-2-negative advanced breast cancer: preliminary correlative results from BOLERO-2. Presented at: The American Society of Clinical Oncology Annual Meeting; May 31-June 4; 2013; Chicago, IL.

35. Treilleux I, Arnedos M, Cropet C, et al. Translational studies within the TAMRAD randomized GINECO trial: evidence for mTORC1 activation marker as a predictive factor for everolimus efficacy in advanced breast cancer. Ann Oncol. 2015;26(1):120-125.

36. Baselga J, Semiglazov V, van Dam P, et al. Phase II randomized study of neoadjuvant everolimus plus letrozole compared with placebo plus letrozole in patients with estrogen receptor-positive breast cancer. J Clin Oncol. 2009;27(16):2630-2637.

37. Bachelot T, Bourgier C, Cropet C, et al. Randomized phase II trial of everolimus in combination with tamoxifen in patients with hormone receptor-positive, human epidermal growth factor receptor 2-negative metastatic breast cancer with prior exposure to aromatase inhibitors: A GINECO study. J Clin Oncol. 2012;30(22):2718-2724.

38. Massarweh S, Romond E, Black EP, et al. A phase II study of combined fulvestrant and everolimus in patients with metastatic estrogen receptor (ER)-positive breast cancer after aromatase inhibitor (AI) failure. Breast Cancer Res Treat. 2014;143(2):325-332.

OncoTargets and Therapy

\section{Publish your work in this journal}

OncoTargets and Therapy is an international, peer-reviewed, open access journal focusing on the pathological basis of all cancers, potential targets for therapy and treatment protocols employed to improve the management of cancer patients. The journal also focuses on the impact of management programs and new therapeutic agents and protocols on
39. Gradishar WJ, Bachelot TD, Saletan S, et al. BOLERO-4: multicenter, open-label, phase II study of everolimus plus letrozole as first-line therapy in ER+, HER2 - metastatic breast cancer. Presented at: The American Society of Clinical Oncology Annual Meeting; May 31-June 4; 2013; Chicago, IL. Abstract TPS661.

40. Munagala R, Aqil F, Gupta RC. Promising molecular targeted therapies in breast cancer. Indian J Pharmacol. 2011;43(3):236-245.

41. Lange CA, Yee D. Killing the second messenger: targeting loss of cell cycle control in endocrine-resistant breast cancer. Endocr Relat Cancer. 2011;18(4):C19-C24.

42. Morikawa A, Henry NL. Palbociclib for the treatment of estrogen receptor-positive, HER2-negative metastatic breast cancer. Clin Cancer Res. 2015;21(16):3591-3596.

43. DeMichele A, Clark AS, Tan KS, et al. CDK 4/6 inhibitor palbociclib (PD0332991) in Rb+ advanced breast cancer: phase II activity, safety, and predictive biomarker assessment. Clin Cancer Res. 2015;21(5):995-1001.

44. Finn RS, Crown JP, Lang I, et al. The cyclin-dependent kinase 4/6 inhibitor palbociclib in combination with letrozole versus letrozole alone as first-line treatment of oestrogen receptor-positive, HER2-negative, advanced breast cancer (PALOMA-1/TRIO-18): a randomised phase 2 study. Lancet Oncol. 2014;16(1):25-35.

45. Pfizer Labs. Ibrance (palbociclib) capsules, for oral use [prescribing information]. New York, NY: Pfizer Labs; February 2015.

46. Turner NC, Ro J, Andre F, et al. Palbociclib in hormone-receptor-positive advanced breast cancer. $N$ Engl J Med. 2015;373(3):209-219.

47. Pollak MN, Schernhammer ES, Hankinson SE. Insulin-like growth factors and neoplasia. Nat Rev Cancer. 2004;4(7):505-518.

48. Nahta R, Yuan LX, Zhang B, Kobayashi R, Esteva FJ. Insulin-like growth factor-I receptor/human epidermal growth factor receptor 2 heterodimerization contributes to trastuzumab resistance of breast cancer cells. Cancer Res. 2005;65(23):11118-11128.

49. Li T, Christos PJ, Sparano JA, et al. Phase II trial of the farnesyltransferase inhibitor tipifarnib plus fulvestrant in hormone receptor-positive metastatic breast cancer: New York Cancer Consortium Trial P6205. Ann Oncol. 2009;20(4):642-647.

50. Johnston SR, Semiglazov VF, Manikhas GM, et al. A phase II, randomized, blinded study of the farnesyltransferase inhibitor tipifarnib combined with letrozole in the treatment of advanced breast cancer after antiestrogen therapy. Breast Cancer Res Treat. 2008;110(2): 327-335.

51. Hanahan D, Folkman J. Patterns and emerging mechanisms of the angiogenic switch during tumorigenesis. Cell. 1996;86(3):353-364.

52. Miles DW, Chan A, Dirix LY, et al. Phase III study of bevacizumab plus docetaxel compared with placebo plus docetaxel for the first-line treatment of human epidermal growth factor receptor 2-negative metastatic breast cancer. J Clin Oncol. 2010;28(20):3239-3247.

53. Robert NJ, Dieras V, Glaspy J, et al. RIBBON-1: randomized, doubleblind, placebo-controlled, phase III trial of chemotherapy with or without bevacizumab for first-line treatment of human epidermal growth factor receptor 2-negative, locally recurrent or metastatic breast cancer. J Clin Oncol. 2011;29(10):1252-1260.

54. Alvarez RH, Kantarjian HM, Cortes JE. The role of Src in solid and hematologic malignancies: development of new-generation Src inhibitors. Cancer. 2006;107(8):1918-1929.

patient perspectives such as quality of life, adherence and satisfaction The manuscript management system is completely online and includes a very quick and fair peer-review system, which is all easy to use. Visit http://www.dovepress.com/testimonials.php to read real quotes from published authors. 